\title{
SIEVED ULTRASPHERICAL POLYNOMIALS
}

\author{
BY
}

\author{
WALEED AL-SALAM, ${ }^{1}$ W. R. ALLAWAY ${ }^{2}$ AND RICHARD ASKEY ${ }^{3}$
}

\begin{abstract}
The continuous $q$-ultraspherical polynomials contain a number of important examples as limiting or special cases. One of these arose in Allaway's Ph.D. thesis. In a previous paper we solved a characterization problem essentially equivalent to Allaway's and showed that these polynomials arose from the $q$-ultraspherical polynomials when $q$ approached a root of unity. A second class of such polynomials is found, and the recurrence relation and orthogonality relation are found for each of these polynomials. The orthogonality is interesting because the weight function has a finite number of zeros in $(-1,1)$. Generating functions and other formulas are also found.
\end{abstract}

1. Introduction. The continuous $q$-ultraspherical polynomials of L. J. Rogers [19] arise as the solution to a number of problems. Their first explicit occurrence as orthogonal polynomials was in papers of Feldheim [15] and Lanzewizky [17]. Here is the problem they solved.

The Fejér-Legendre polynomials $\left\{p_{n}(x)\right\}_{n=0}^{\infty}, x=\cos \theta$, are defined by

$$
|f(r \exp (i \theta))|^{2}=\sum_{n=0}^{\infty} r^{n} \sum_{k=0}^{n} a_{k} a_{n-k} \cos (n-2 k) \theta=\sum_{n=0}^{\infty} p_{n}(x) r^{n},
$$

where $a_{n}$ is real and

$$
f(z)=\sum_{n=0}^{\infty} a_{n} z^{n}
$$

converges in the neighbourhood of $z=0$.

If the Fejér-Legendre polynomials are orthogonal, Feldheim and Lanzewizky showed they can be rescaled to satisfy

$$
2 x\left(1-\beta q^{n}\right) C_{n}(x ; \beta \mid q)=\left(1-q^{n+1}\right) C_{n+1}(x ; \beta \mid q)+\left(1-\beta^{2} q^{n-1}\right) C_{n-1}(x ; \beta \mid q)
$$

Received by the editors March 4, 1983.

1980 Mathematics Subject Classification. Primary 33A65.

Key words and phrases. Sieved ultraspherical polynomials of first and second kind, continuous $q$-ultraspherical polynomials, Fejer-Legendre polynomials, orthogonal polynomials, ultraspherical polynomials, Tchebycheff polynomials, recurrence relation, weight function, generating function, product linearization, connection coefficient.

\footnotetext{
'Supported in part by N.S.E.R.C. Grant A2975.

${ }^{2}$ Supported in part by N.S.E.R.C. Grant A8721 and the President's N.S.E.R.C. Fund (Lakehead University).

${ }^{3}$ Supported in part by N.S.F. Grant MCS- 8101568 .
} 
for $n \geqslant 1$, where $C_{0}(x ; \beta \mid q)=1, C_{1}(x ; \beta \mid q)=2 x(1-\beta) /(1-q)$, and $|q|<1$, or a limiting case of this recurrence relation. They knew the polynomials were orthogonal for appropriate choices of $\beta$ and $q$ since any set of polynomials that satisfies

$$
x p_{n}(x)=A_{n} p_{n+1}(x)+B_{n} p_{n}(x)+C_{n} p_{n-1}(x),
$$

$p_{-1}(x)=0, p_{0}(x)=1$, with $A_{n} C_{n+1}>0, A_{n}, B_{n}$ and $C_{n+1}$ real for $n=0,1, \ldots$, must be orthogonal with respect to a positive measure (see $[14$, p. 21]). However, they were unable to find the orthogonality relation explicitly. When $-1<\beta<1$, and $-1<q$ $<1$ the orthogonality is

$$
\begin{aligned}
\int_{-1}^{1} C_{n}(x ; \beta \mid q) C_{m}(x ; \beta \mid q) \prod_{j=0}^{\infty}\left[\frac{1-2\left(2 x^{2}-1\right) q^{j}+q^{2 j}}{1-2\left(2 x^{2}-1\right) \beta q^{j}+\beta^{2} q^{2 j}}\right] \frac{d x}{\left(1-x^{2}\right)^{1 / 2}} \\
= \begin{cases}0 & \text { if } m \neq n \\
\frac{2 \pi(1-\beta)\left(\beta^{2} ; q\right)_{n}(\beta ; q)_{\infty}(\beta q ; q)_{\infty}}{\left(1-\beta q^{n}\right)(q ; q)_{n}\left(\beta^{2} ; q\right)_{\infty}(q ; q)_{\infty}} & \text { if } m=n .\end{cases}
\end{aligned}
$$

Unless otherwise stated $|q|<1$ will be assumed. Then,

$$
\begin{gathered}
(a ; q)_{\infty}:=\prod_{n=0}^{\infty}\left(1-a q^{n}\right), \\
(a ; q)_{n}:=(a ; q)_{\infty} /\left(a q^{n} ; q\right)_{\infty} .
\end{gathered}
$$

See [19 or 7] for the recurrence relation and [6, 7, 8 or 9] for proofs of the orthogonality of the continuous $q$-ultraspherical polynomials $\left\{C_{n}(x ; \beta \mid q)\right\}_{n=0}^{\infty}$. The generating function (1.1) for these polynomials is

$$
\sum_{n=0}^{\infty} C_{n}(\cos \theta ; \beta \mid q) r^{n}=\frac{(\beta r \exp (i \theta) ; q)_{\infty}(\beta r \exp (-i \theta) ; q)_{\infty}}{(r \exp (i \theta) ; q)_{\infty}(r \exp (-i \theta) ; q)_{\infty}}
$$

and the $q$-binomial theorem can be used to sum series (1.2)

$$
\sum_{n=0}^{\infty} \frac{(\beta ; q)_{n} z^{n}}{(q ; q)_{n}}=\frac{(\beta z ; q)_{\infty}}{(z ; q)_{\infty}}
$$

See $[3,5,11,13$ or 20] for simple proofs of (1.9).

A second problem that leads to these polynomials was solved by Allaway [2]. He found all orthogonal polynomials $\left\{p_{n}(x)\right\}_{n=0}^{\infty}$ that have expansions of the form

$$
v(x) p_{n}(x)=\sum_{k=0}^{\infty} a_{k} b_{n+k} U_{n+2 k}(x)
$$

where the Tchebycheff polynomials of the second kind, $\left\{U_{n}(x)\right\}_{n=0}^{\infty}$, are defined by

$$
U_{n}(\cos \theta)=\sin (n+1) \theta / \sin \theta \text {. }
$$

The answer to this question turned out to be the continuous $q$-ultraspherical polynomials with $|\beta|<1,|q|<1$, and some appropriate limiting cases. The reason for considering this problem came from one of the limiting cases. When $\beta=q^{\lambda}$, divide both sides of $(1.3)$ by $(1-q)$ and let $q \rightarrow 1$. The result is

$$
2(n+\lambda) x C_{n}^{\lambda}(x)=(n+1) C_{n+1}^{\lambda}(x)+(n+2 \lambda-1) C_{n-1}^{\lambda}(x),
$$


$n \geqslant 1, C_{0}^{\lambda}(x)=1, C_{1}^{\lambda}(x)=2 \lambda x .\left\{C_{n}^{\lambda}(x)\right\}_{n=0}^{\infty}$ are the ultraspherical polynomials whose orthogonality relation is

$$
\int_{-1}^{1} C_{n}^{\lambda}(x) C_{m}^{\lambda}(x)\left(1-x^{2}\right)^{\lambda-1 / 2} d x=\frac{2^{-2 \lambda} \pi(2 \lambda)_{n} \Gamma(2 \lambda+1)}{(n+\lambda) n ! \Gamma(\lambda) \Gamma(\lambda+1)} \delta_{m, n} .
$$

Here $(a)_{n}$ is defined by

$$
(a)_{n}=\Gamma(n+a) / \Gamma(a) .
$$

Szegö showed that for $n \geqslant 0$,

$$
\left(1-x^{2}\right)^{\lambda-1} \frac{C_{n}^{\lambda}(x)}{C_{n}^{\lambda}(1)}=\frac{2 \Gamma(\lambda+(1 / 2))}{\Gamma(1 / 2) \Gamma(\lambda+1)} \sum_{k=0}^{\infty} \frac{(1-\lambda)_{k}(n+k) !}{k !(\lambda+1)_{n+k}} U_{n+2 k}(x),
$$

which has the form (1.10).

A problem essentially equivalent to the one solved in Allaway's Ph.D. thesis [2] was solved in [1]. In both of these treatments not only do the continuous $q$-ultraspherical polynomials arise and their limiting case the ultraspherical polynomials, but a second limiting case also arises. In [1] it is pointed out that this limiting case is obtained when $\beta=s^{\lambda k}, q=s \omega_{k}$ and $s \rightarrow 1$. Here $\omega_{k}=\exp (2 \pi i / k)$.

Both of the representations given by (1.1) and (1.10) for the continuous $q$-ultraspherical polynomials are special cases of more general identities between these polynomials. Rogers [19] (see [7] for another proof) showed that for $n \geqslant 0$

$$
\begin{aligned}
C_{n}(x ; \gamma \mid q) & =\sum_{k=0}^{\lfloor n / 2\rfloor} a(k, n) C_{n-2 k}(x ; \beta \mid q), \\
a(k, n) & =\frac{\beta^{k}(\gamma / \beta ; q)_{k}(\gamma ; q)_{n-k}\left(1-\beta q^{n-2 k}\right)}{(q ; q)_{k}(\beta q ; q)_{n-k}(1-\beta)} .
\end{aligned}
$$

Since for $n \geqslant 1$

$$
\lim _{\beta \rightarrow 1} \frac{1-q^{n}}{1-\beta} C_{n}(\cos \theta ; \beta \mid q)=2 \cos n \theta
$$

this extends

$$
C_{n}(\cos \theta ; \beta \mid q)=\sum_{k=0}^{n} \frac{(\beta ; q)_{k}(\beta ; q)_{n-k}}{(q ; q)_{k}(q ; q)_{n-k}} \cos (n-2 k) \theta .
$$

This is the formula obtained when (1.8) and (1.9) are combined as (1.1). The extension of $(1.10)$ is

$$
v(x) C_{n}(x ; \beta \mid q)=\sum_{k=0}^{\infty} d(k, n) C_{n+2 k}(x ; \gamma \mid q),
$$

with

$$
\begin{gathered}
d(k, n)=\frac{\beta^{k}(\gamma / \beta ; q)_{k}(q ; q)_{n+2 k}\left(\beta^{2} ; q\right)_{n}(\gamma ; q)_{n+k}\left(1-\gamma q^{n+2 k}\right)}{(q ; q)_{k}\left(\gamma^{2} ; q\right)_{n+2 k}(q ; q)_{n}(\beta q ; q)_{n+k}(1-\gamma)} A, \\
A=\frac{\left(\gamma^{2} ; q\right)_{\infty}(\beta ; q)_{\infty}(\beta q ; q)_{\infty}}{(\gamma ; q)_{\infty}(\gamma q ; q)_{\infty}\left(\beta^{2} ; q\right)_{\infty}}
\end{gathered}
$$


and

$$
v(x)=\prod_{j=0}^{\infty}\left[\frac{1-2\left(2 x^{2}-1\right) \gamma q^{j}+\gamma^{2} q^{2 j}}{1-2\left(2 x^{2}-1\right) \beta q^{j}+\beta^{2} q^{2 j}}\right]
$$

(see [7]). (1.19) is a consequence of the orthogonality relation (1.5) and (1.16). When $\gamma=q$

$$
C_{n}(x ; q \mid q)=U_{n}(x)
$$

and (1.19) becomes

$$
w(x) C_{n}(x ; \beta \mid q)=\sum_{k=0}^{\infty} a_{k} b_{n+k} d_{n} U_{n+2 k}(x),
$$

with $a_{k}=\beta^{k}(q / \beta ; q)_{k} /(q ; q)_{k}, b_{k}=(q ; q)_{k} /(\beta q ; q)_{k}$ and

$$
d_{n}=\frac{\left(\beta^{2} ; q\right)_{n}(\beta ; q)_{\infty}(\beta q ; q)_{\infty}}{(q ; q)_{n}\left(\beta^{2} ; q\right)_{\infty}(q ; q)_{\infty}} .
$$

In $\$ 2$ we will give the orthogonality relation and recurrence relation for the limiting case that Allaway considered in [2]. In $\$ 3$ we will treat the other limiting case when $q$ approaches a root of unity and the polynomials are orthogonal with respect to a positive measure. This case was not mentioned by Feldheim [15] or Lanzewizky [17] but it is contained in their solutions as a limiting case. The rest of the paper will contain some further facts about these two classes of orthogonal polynomials.

2. Sieved ultraspherical polynomials of the first kind. Renormalize the continuous $q$-ultraspherical polynomials by setting

$$
C_{n}(x ; \beta \mid q)=\left(\beta^{2} ; q\right)_{n} c_{n}(x ; \beta \mid q) /(q ; q)_{n} .
$$

The recurrence relation becomes for $n \geqslant 0$

$$
2 x\left(1-\beta q^{n}\right) c_{n}(x ; \beta \mid q)=\left(1-\beta^{2} q^{n}\right) c_{n+1}(x ; \beta \mid q)+\left(1-q^{n}\right) c_{n-1}(x ; \beta \mid q),
$$

where $c_{-1}(x ; \beta \mid q)=0$ and $c_{0}(x ; \beta \mid q)=1$. Set $\beta=s^{\lambda k}, q=s \exp (2 \pi i / k)=s \omega_{k}$, divide by $\left(1-s \omega_{k}^{n}\right)$ and take the limit as $s \rightarrow 1$, to obtain the resulting polynomial set $\left\{c_{n}^{\lambda}(x ; k)\right\}$ which has a recurrence relation of the form

$$
\left\{\begin{array}{l}
2 x c_{n}^{\lambda}(x ; k)=c_{n+1}^{\lambda}(x ; k)+c_{n-1}^{\lambda}(x ; k), \quad n \neq m k, \\
2 x(m+\lambda) c_{m k}^{\lambda}(x ; k)=(m+2 \lambda) c_{m k+1}^{\lambda}(x ; k)+m c_{m k-1}^{\lambda}(x ; k),
\end{array}\right.
$$

where $c_{0}^{\lambda}(x ; k)=1 ; c_{1}^{\lambda}(x ; k)=x$. Note that $c_{n}^{\lambda}(x ; 1)=n ! C_{n}^{\lambda}(x) /(2 \lambda)_{n}$. A natural name for these polynomials is the sieved ultraspherical polynomials, for a sieve has operated on the recurrence relation for ultraspherical polynomials after they have been renormalized and $\lambda$ has been replaced by $\lambda k$. There is a second kind of sieved ultraspherical polynomial (see $\S 3$ ), so the polynomial set $\left\{c_{n}^{\lambda}(x ; k)\right\}_{n=0}^{\infty}$ defined by the recurrence relation (2.3) will be called sieved ultraspherical polynomials of the first kind. Allaway discovered the recurrence relation (2.3) in [2], but did not study these polynomials further. In particular he did not work out formula (1.10) explicitly. 
Once this series is summed the orthogonality relation can be obtained formally. For,

$$
\begin{aligned}
\int_{-1}^{1} p_{n}(x) U_{j}(x) v(x) & \left(1-x^{2}\right)^{1 / 2} d x \\
= & \sum_{k=0}^{\infty} a_{k} b_{n+k} \int_{-1}^{1} U_{j}(x) U_{n+2 k}(x)\left(1-x^{2}\right)^{1 / 2} d x=0,
\end{aligned}
$$

if $j<n$, so $p_{n}(x)$ is orthogonal to all polynomials of degree less than $n$ with respect to $v(x)\left(1-x^{2}\right)^{1 / 2}$ on $[-1,1]$. If the series (1.10) converges rapidly enough this argument is correct. Unfortunately, the series (1.10) does not always converge, and if it does there may be problems in justifying the term by term integration in (2.4). There are other ways to obtain the orthogonality relation. Rewrite (1.5) as

$$
\begin{gathered}
\int_{-1}^{1} c_{n}(x ; \beta \mid q) c_{m}(x ; \beta \mid q) \prod_{j=0}^{\infty}\left[\frac{1-2\left(2 x^{2}-1\right) q^{j}+q^{2 j}}{1-2\left(2 x^{2}-1\right) \beta q^{j}+\beta^{2} q^{2 j}}\right] \frac{d x}{\sqrt{1-x^{2}}}=h_{n} \delta_{n, m}, \\
h_{n}=\frac{2 \pi(1-\beta)(q ; q)_{n}(\beta ; q)_{\infty}(\beta q ; q)_{\infty}}{\left(1-\beta q^{n}\right)\left(\beta^{2} ; q\right)_{n}\left(\beta^{2} ; q\right)_{\infty}(q ; q)_{\infty}} .
\end{gathered}
$$

The factors in $h_{n}$ that depend on $n$ can be rewritten as

$$
\frac{(1-\beta)(q ; q)_{n}}{\left(1-\beta q^{n}\right)\left(\beta^{2} ; q\right)_{n}}=\frac{(\beta ; q)_{n}(q ; q)_{n}}{\left(\beta^{2} ; q\right)_{n}(\beta q ; q)_{n}}
$$

The remaining factors in $h_{n}$ that depend on $q$ are

$$
\frac{(\beta ; q)_{\infty}(\beta q ; q)_{\infty}}{\left(\beta^{2} ; q\right)_{\infty}(q ; q)_{\infty}}=\prod_{n=0}^{\infty} \frac{\left(1-s^{n+\lambda k} \omega_{k}^{n}\right)\left(1-s^{n+\lambda k+1} \omega_{k}^{n+1}\right)}{\left(1-s^{n+2 \lambda k} \omega_{k}^{n}\right)\left(1-s^{n+1} \omega_{k}^{n+1}\right)}
$$

There are two sets of factors to consider when evaluating the limit as $s \rightarrow 1$ of the right-hand side of (2.7). First, consider all the factors that have $\omega_{k}^{n}=1$. These are

$$
\prod_{m=0}^{\infty} \frac{\left(1-t^{m+\lambda}\right)\left(1-t^{m+\lambda+1}\right)}{\left(1-t^{m+2 \lambda}\right)\left(1-t^{m+1}\right)}=\frac{\Gamma_{t}(2 \lambda)}{\Gamma_{t}(\lambda) \Gamma_{t}(\lambda+1)},
$$

where $s^{k}=t$ and the $q$-gamma function $\Gamma_{q}(x)$ is defined by

$$
\Gamma_{q}(x)=(q ; q)_{\infty}(1-q)^{1-x} /\left(q^{x} ; q\right)_{\infty} .
$$

The limit of these factors can be computed when $s \rightarrow 1$ because

$$
\lim _{q \rightarrow 1} \Gamma_{q}(x)=\Gamma(x)
$$

(see [4]).

The $q$-binomial theorem, equation (1.9), can be used to find the limit of the remaining factors on the right-hand side of (2.7). When $\beta=q^{\alpha}$ and $z=x q^{\gamma}$ we obtain

$$
\begin{aligned}
\lim _{q \rightarrow 1} \frac{\left(q^{\alpha+\gamma} x ; q\right)_{\infty}}{\left(q^{\gamma} x ; q\right)_{\infty}} & =\lim _{q \rightarrow 1} \sum_{n=0}^{\infty} \frac{\left(q^{\alpha} ; q\right)_{n}}{(q ; q)_{n}}\left(q^{\gamma} x\right)^{n} \\
& =\sum_{n=0}^{\infty} \frac{(\alpha)_{n}}{n !} x^{n}=(1-x)^{-\alpha}
\end{aligned}
$$


when $|x|<1$, and by analytic continuation this holds in the complex plane cut on $[1, \infty)$. Use a sieve on the right-hand side of (2.7) and take the limit as $s \rightarrow 1$ to obtain

$$
\prod_{j=1}^{k-1}(1-\exp (2 \pi i j / k))^{\lambda}(1-\exp (2 \pi i j / k))^{-\lambda}=1
$$

Thus,

$$
\lim _{s \rightarrow 1} \frac{\left(s^{\lambda k} ; s \omega_{k}\right)_{\infty}\left(s^{\lambda k+1} \omega_{k} ; s \omega_{k}\right)_{\infty}}{\left(s^{2 \lambda k} ; s \omega_{k}\right)_{\infty}\left(s \omega_{k} ; s \omega_{k}\right)_{\infty}}=\frac{\Gamma(2 \lambda)}{\Gamma(\lambda) \Gamma(\lambda+1)}
$$

and

$$
h_{n}=\frac{2 \pi(1)_{\lfloor n / k\rfloor}(\lambda)_{\lceil n / k\rceil}}{(\lambda+1)_{\lfloor n / k\rfloor}(2 \lambda)_{\lceil n / k\rceil}} \frac{\Gamma(2 \lambda)}{\Gamma(\lambda) \Gamma(\lambda+1)},
$$

where the roof and floor functions are defined by:

$\lceil a\rceil=$ smallest integer greater than or equal to $a$,

$\lfloor a\rfloor=$ largest integer less than or equal to $a$.

The weight function is

$$
\begin{aligned}
w_{s}(x) & =\left(1-x^{2}\right)^{-1 / 2} \prod_{n=0}^{\infty}\left[\frac{1-2\left(2 x^{2}-1\right) s^{n} \omega_{k}^{n}+s^{2 n} \omega_{k}^{2 n}}{1-2\left(2 x^{2}-1\right) s^{n+\lambda k} \omega_{k}^{n}+s^{2 n+2 \lambda k} \omega_{k}^{2 n}}\right] \\
& =\left(1-x^{2}\right)^{-1 / 2} \prod_{n=0}^{\infty} \prod_{j=0}^{k-1}\left[\frac{1-2\left(2 x^{2}-1\right) s^{n k+j} \omega_{k}^{j}+s^{2 n k+2 j} \omega_{k}^{2 j}}{1-2 j\left(2 x^{2}-1\right) s^{(n+\lambda) k+j} \omega_{k}^{j}+s^{2(n+\lambda) k+2 j} \omega_{k}^{2 j}}\right] .
\end{aligned}
$$

Then,

$$
w(x)=\lim _{s \rightarrow 1} w_{s}(x)=\left(1-x^{2}\right)^{-1 / 2} \prod_{j=0}^{k-1}\left[1-2\left(2 x^{2}-1\right) \omega_{k}^{-j}+\omega_{k}^{-2 j}\right]^{\lambda} .
$$

But,

$$
w(x)=\left(1-x^{2}\right)^{-1 / 2} \prod_{j=0}^{k-1}\left[1-2\left(2 x^{2}-1\right) \omega_{k}^{-j}+\omega_{k}^{-2 j}\right]^{\lambda},
$$

since $\omega_{k}^{j}=\exp (2 \pi i j / k)=\omega_{k}^{-(k-j)}$. Observe that

$$
\begin{aligned}
& {\left[1-2\left(2 x^{2}-1\right) \omega_{k}^{j}+\omega_{k}^{2 j}\right]\left[1-2\left(2 x^{2}-1\right) \omega_{k}^{-j}+\omega_{k}^{-2 j}\right]} \\
& =\left[2 \cos (2 \pi j / k)-2\left(2 x^{2}-1\right)\right]^{2} .
\end{aligned}
$$

Thus,

$$
\begin{aligned}
{[w(x)]^{2} } & =\left(1-x^{2}\right)^{-1}\left(4-4 x^{2}\right)^{2 \lambda}\left[\prod_{j=1}^{k-1}\left(2+2 \cos (2 \pi j / k)-4 x^{2}\right)^{2}\right]^{\lambda} \\
& =\left(1-x^{2}\right)^{-1}\left[\prod_{j=0}^{k-1}\left(4 \cos ^{2}(\pi j / k)-4 x^{2}\right)^{2}\right]^{\lambda}
\end{aligned}
$$


This gives

$$
w(x)=\left(1-x^{2}\right)^{-1 / 2} 2^{2 \lambda k} \prod_{j=0}^{k-1}\left|\cos (2 \pi j / k)-x^{2}\right|^{\lambda} .
$$

We will multiply this function by $2^{-2 \lambda}$ to simplify it (see (2.14)). This is the weight function when it is integrable, i.e. when $\lambda>-1 / 2$.

The careful reader will be aware of two problems that were ignored in this formal calculation. First, the orthogonality relation (1.5) was only claimed for $-1<\beta<1$, and this means $\lambda>0$ rather than $\lambda>-1 / 2$. When $1<\beta<q^{-1 / 2}, 0<q<1$, there are two discrete masses that have to be added. They disappear in the limit. Second, the orthogonality relation was only claimed to hold when the weight function is positive. When $|q|<1$ this forces $-1<q<1$. We took $q=s \exp (2 \pi i / k)$, which is not allowed. One way around both of these problems is to use the more general complex measure and contour integral given in [9]. We will not give the details here since there are more general sieved polynomials, sieved Jacobi polynomials, that contain the sieved ultraspherical polynomials as special cases. The details of obtaining their orthogonality from the complex orthogonality in [9] will be given in another paper. The reason for considering the sieved ultraspherical polynomials separately is that they have a number of very nice formulas that do not extend to the sieved Jacobi polynomials, or if they extend the extensions are much more complicated.

The integral of $w(x)$ exists when $\lambda>-1 / 2$. We would like to show that the polynomials $\left\{c_{n}^{\lambda}(x ; k)\right\}_{n=0}^{\infty}$ are orthogonal with respect to a positive measure exactly when the integral of $w(x)$ exists. If $\lambda>-1 / 2$, then the coefficients in (1.4) are given by $a_{n}=c_{n}=1 / 2$, when $n \neq m k$ and $a_{m k}=(m+2 \lambda) /(2 m+2 \lambda), c_{m k}=$ $m /(2 m+2 \lambda)$. Therefore $a_{n} c_{n+1}>0, n=0,1,2, \ldots$, when $\lambda>-1 / 2$ and thus by (1.4) $\left\{c_{n}^{\lambda}(x ; k)\right\}_{n=0}^{\infty}$ is orthogonal with respect to a positive measure.

The above calculations can be summarized as followed.

THEOREM 1. Let $k$ be any positive integer and $\lambda>-1 / 2$. Define the polynomials $c_{n}^{\lambda}(x ; k)$ by the recurrence relation $(2.3)$. Then

$$
\int_{-1}^{1} c_{n}^{\lambda}(x ; k) c_{m}^{\lambda}(x ; k) w(x) d x=\delta_{m, n} h_{n}
$$

with

$$
\begin{aligned}
w(x) & =2^{2 \lambda(k-1)}\left(1-x^{2}\right)^{-1 / 2} \prod_{j=0}^{k-1}\left|x^{2}-\cos ^{2}(\pi j / k)\right|^{\lambda} \\
& =\left(1-x^{2}\right)^{\lambda-1 / 2}\left|U_{k-1}(x)\right|^{2 \lambda}
\end{aligned}
$$

and

$$
h_{n}=\frac{(\lambda)_{\lceil n / k\rceil}(1)_{\lfloor n / k\rfloor} \Gamma(1 / 2) \Gamma(\lambda+1 / 2) l}{(2 \lambda)_{\lceil n / k\rceil}(\lambda+1)_{\lfloor n / k\rfloor} \Gamma(\lambda+1)} .
$$

One surprising special case occurs for $m, n \leqslant k$. If $x=\cos \theta$, then for $j=$ $0,1, \ldots, k$,

$$
c_{j}^{\lambda}(x ; k)=T_{j}(x)=\cos j \theta ;
$$


we have

$$
\begin{aligned}
& \int_{-1}^{1} T_{m}(x) T_{n}(x)\left|U_{k-1}(x)\right|^{2 \lambda}\left(1-x^{2}\right)^{\lambda-1 / 2} d x \\
& = \begin{cases}0, & \text { if } 0 \leqslant m \neq n \leqslant k, \\
\Gamma(1 / 2) \Gamma(\lambda+1 / 2) / \Gamma(\lambda+1)=h_{0} & \text { if } m=n=0, \\
h_{0} / 2 & \text { if } 1 \leqslant m=n \leqslant k-1, \\
h_{0} / 2(\lambda+1) & \text { if } m=n=k .\end{cases}
\end{aligned}
$$

Multiply both sides of (2.17) by $\lambda+1 / 2$ and let $\lambda \rightarrow 1 / 2$. Since $(\lambda+1 / 2) h_{0} \rightarrow 1$ as $\lambda \rightarrow-1 / 2$, the measure does not vanish. It becomes a finite number of point masses located at $x= \pm 1$ and at the zeros of $U_{k-1}(x)$, and the resulting orthogonality is equivalent to a well-known finite orthogonality for $\cos n \theta$.

$$
\begin{aligned}
\frac{1}{2 k}+ & \frac{1}{k} \sum_{j=1}^{k-1} \cos \frac{m \pi j}{k} \cos \frac{n \pi j}{k}+\frac{1}{2 k} \cos m \pi \cos n \pi \\
& = \begin{cases}0, & \text { if } 0 \leqslant m \neq n \leqslant k, \\
1, & \text { if } m=n=0 \text { or } m=n=k, \\
1 / 2, & \text { if } 1 \leqslant m=n \leqslant k-1 .\end{cases}
\end{aligned}
$$

Since $c_{j}^{\lambda}(1 ; k)=1$, for $0 \leqslant j \leqslant k$, this suggests looking at $c_{j}^{\lambda}(1 ; k)$ for other $j$ 's. A simple induction using (2.3) shows that for $j \geqslant 0$

$$
c_{j}^{\lambda}(1 ; k)=1 \text {. }
$$

3. Sieved ultraspherical polynomials of the second kind. To find the other kind of sieved ultraspherical polynomials put $\beta=s^{\lambda k+1} \omega_{k}, q=s \omega_{k}$ in (1.3), divide by $\left(1-s \omega_{k}^{n+1}\right)$ and let $s \rightarrow 1$. Denote the resulting polynomials by $B_{n}^{\lambda}(x ; k)$. Their recurrence relation is

$$
\begin{aligned}
& 2 x B_{n}^{\lambda}(x ; k)=B_{n+1}^{\lambda}(x ; k)+B_{n-1}^{\lambda}(x ; k), \quad n+1 \neq m k, \\
& 2 x(m+\lambda) B_{m k-1}^{\lambda}(x ; k)=m B_{m k}^{\lambda}(x ; k)+(m+2 \lambda) B_{m k-2}^{\lambda}(x ; k),
\end{aligned}
$$

where $B_{0}^{\lambda}(x ; k)=1 ; B_{1}^{\lambda}(x ; k)=2 x$, if $k \geqslant 2 ; B_{1}^{\lambda}(x ; 1)=2(\lambda+1) x$. Note that $B_{n}^{\lambda}(x ; 1)=C_{n}^{\lambda+1}(x)$.

The formal calculations in $\$ 2$ can be done in this case and lead to the correct results. The resulting orthogonality relation follows.

THEOREM 2. Let $k$ be any positive integer and $\lambda>-1 / 2$. Define the polynomials $B_{n}^{\lambda}(x ; k)$ by the recurrence relation (3.1). Then

$$
\int_{-1}^{1} B_{m}^{\lambda}(x ; k) B_{n}^{\lambda}(x ; k) w(x) d x=\delta_{m, n} h_{n}
$$

with

$$
\begin{aligned}
w(x) & =2^{2 \lambda(k-1)}\left(1-x^{2}\right)^{1 / 2} \prod_{j=0}^{k-1}\left|x^{2}-\cos ^{2}(\pi j / k)\right|^{\lambda} \\
& =\left(1-x^{2}\right)^{\lambda+1 / 2}\left|U_{k-1}(x)\right|^{2 \lambda}
\end{aligned}
$$


and

$$
h_{n}=\frac{(\lambda+1)_{\lfloor n / k\rfloor}(2 \lambda+1)_{\lfloor(n+1) / k\rfloor}}{2(1)_{\lfloor n / k\rfloor}(\lambda+1)_{\lfloor(n+1) / k\rfloor}} \frac{\Gamma(1 / 2) \Gamma(\lambda+1 / 2)}{\Gamma(\lambda+1)} .
$$

In this case

$$
B_{n}^{\lambda}(x ; k)=U_{n}(x), \quad n=0,1, \ldots, k-1,
$$

and

$$
B_{k}^{\lambda}(x ; k)==2 x(\lambda+1) U_{k-1}(x)-(2 \lambda+1) U_{k-2}(x)
$$

Orthogonality with respect to a positive measure holds when $\lambda>-1 / 2$. When $\lambda \rightarrow-1 / 2$ a discrete orthogonality arises, but this time the point masses at $x= \pm 1$ do not appear. The discrete orthogonality is equivalent to a known discrete orthogonality, namely

$$
\frac{2}{k} \sum_{j=1}^{k-1} \sin \frac{n \pi j}{k} \sin \frac{m \pi j}{k}=\delta_{m, n}, \quad 1 \leqslant m, n \leqslant k-1 .
$$

The analogue of $(2.17)$ is

$$
\int_{-1}^{1} U_{m}(x) U_{n}(x)\left|U_{k-1}(x)\right|^{2 \lambda}\left(1-x^{2}\right)^{\lambda+1 / 2} d x=h_{m} \delta_{m, n}, \quad 0 \leqslant m, n \leqslant k-1,
$$

with $h_{m}=\Gamma(1 / 2) \Gamma(\lambda+1 / 2) / 2 \Gamma(\lambda+1)$ for $n=0,1,2, \ldots, k-2$ and $h_{k-1}=$ $(2 \lambda+1) h_{0} /(\lambda+1)$.

The reason for the notation $B_{n}^{\lambda}(x ; k)$ rather than $C_{n}^{\lambda}(x ; k)$ is that the orthogonality relation in Theorem 2 does not reduce to that of $C_{n}^{\lambda}(x)$ when $k=1$, but to that of $C_{n}^{\lambda+1}(x)$. It would be natural to define $C_{n}^{\lambda}(x ; k)=B_{n}^{\lambda-1}(x ; k)$, but the resulting formulas would be a bit more complicated to write. The notation adopted should be as simple as possible, as close to previous notation as possible, and as suggestive as possible. When it is not possible to have all of these, simplicity is a natural choice.

4. Generating functions and related results. The generating function (1.8) is very useful. To find the resulting generating function for the sieved ultraspherical polynomials of the second kind $\left\{B_{n}^{\lambda}(x ; k)\right\}_{n=0}^{\infty}$ start with $f(z)$ defined by the $q$-binomial theorem (1.9), take $\beta=s^{\lambda k+1} \omega_{k}, q=s \omega_{k}$ and let $s \rightarrow 1$. The results are

$$
a_{n k+j}=\lim _{s \rightarrow 1} \frac{(\beta ; q)^{n k+j}}{(q ; q)_{n k+j}}=(\lambda+1)_{n} / n !
$$

for $j=0,1,2, \ldots, k-1$. Then

$$
f(z)=\sum_{n=0}^{\infty} \frac{(\lambda+1)_{n} z^{k}}{n !} \cdot \frac{1-z^{k}}{1-z}=\left(1-z^{k}\right)^{-\lambda}(1-z)^{-1} .
$$

The generating function for $B_{n}^{\lambda}(x ; k)$ is

$$
\sum_{n=0}^{\infty} B_{n}^{\lambda}(x ; k) r^{n}=\left(1-2 x r+r^{2}\right)^{-1}\left(1-2 T_{k}(x) r^{k}+r^{2 k}\right)^{-\lambda} \text {. }
$$


An explicit representation can be found from (4.3)

$$
B_{n}^{\lambda}(\cos \theta ; k)=\sum_{0 \leqslant j \leqslant n / k} U_{n-k j}(\cos \theta) C_{j}^{\lambda}(\cos k \theta) .
$$

Another representation is

$$
B_{n}(\cos \theta ; k)=\sum_{j=0}^{n} a_{j} a_{n-j} \cos (n-2 j) \theta,
$$

with $a_{j}$ 's given by (4.1). Since $a_{j}>0$, when $\lambda>-1$ and $|\cos \theta| \leqslant \cos 0=1$, we have for $-1 \leqslant x \leqslant 1$ and $\lambda>-1$

$$
\left|B_{n}^{\lambda}(\cos \theta ; k)\right| \leqslant B_{n}^{\lambda}(1 ; k) .
$$

For the sieved ultraspherical polynomials of the first kind this argument leads to a partial generating function. The results are

$$
f(z)=\left(1-z^{k}\right)^{-\lambda}
$$

and

$$
\sum_{n=0}^{\infty} \frac{(2 \lambda)_{n}}{n !} c_{n k}^{\lambda}(x ; k) r^{n k}=\left(1-2 T_{k}(x) r^{k}+r^{2 k}\right)^{-\lambda}
$$

There is a full generating function and it can be found as follows. Use the generating function (1.8) to go from

$$
\sum_{n=0}^{\infty} C_{n}(\cos \theta ; \beta \mid q) r^{n}-\beta \sum_{n=0}^{\infty} C_{n}(\cos \theta ; \beta \mid q)(q r)^{n}
$$

to

$$
\sum_{n=0}^{\infty} \frac{\left(1-\beta q^{n}\right)}{(1-\beta)} C_{n}(\cos \theta ; \beta \mid q) r^{n}=\frac{\left(1-\beta r^{2}\right)\left(\beta q r e^{i \theta} ; q\right)_{\infty}\left(\beta q r e^{-i \theta} ; q\right)_{\infty}}{\left(r e^{i \theta} ; q\right)_{\infty}\left(r e^{-i \theta} ; q\right)_{\infty}}
$$

This leads to

$$
\sum_{n=0}^{\infty} \alpha_{n} c_{n}^{\lambda}(x ; k) r^{n}=\frac{1-r^{2}}{\left(1-2 x r+r^{2}\right)\left(1-2 T_{k}(x) r^{k}+r^{2 k}\right)^{\lambda}},
$$

with $\alpha_{n}$ given by

$$
\alpha_{n}=\frac{(\lambda+1)_{\lfloor n / k\rfloor}(2 \lambda)_{\lceil n / k\rceil}}{(1)_{\lfloor n / k\rfloor} \lambda_{\lceil n / k\rceil}}
$$

Formulas (4.3) and (4.10) imply

$$
\alpha_{n} c_{n}^{\lambda}(x ; k)=B_{n}^{\lambda}(x ; k)-B_{n-2}^{\lambda}(x ; k), \quad n \geqslant 2 .
$$

When (4.4) and (4.12) are combined the result is

$$
\alpha_{n} c_{n}^{\lambda}(\cos \theta ; k)=\sum_{0 \leqslant j \leqslant n / k} a_{n-j k} T_{n-j k}(\cos \theta) C_{j}^{\lambda}(\cos k \theta),
$$

where $a_{0}=1$ and $a_{n}=2$ for $n \geqslant 1$. By using the generating functions for $T_{n}(x)$ and $C_{n}^{\lambda}(x),(4.13)$ also follows from (4.10). 
From (4.10)

$$
\begin{aligned}
& \sum_{n=0}^{\infty} \alpha_{n} c_{n}^{\lambda}(\cos \theta ; k) r^{n} \\
& =\left[1+2 \sum_{j=1}^{\infty} r^{j} \cos j \theta\right]\left[\sum_{n=0}^{\infty} r^{n k} \sum_{j=0}^{n} \frac{(\lambda)_{j}(\lambda)_{n-j}}{j !(n-j) !} \cos (n-2 j) k \theta\right],
\end{aligned}
$$

but

$$
\cos j \theta \cos m \theta=\frac{1}{2}[\cos (j-m) \theta+\cos (j+m) \theta]
$$

so

$$
c_{n}^{\lambda}(\cos \theta ; k)=\sum_{j=0}^{n} f_{j}(\lambda) \cos j \theta,
$$

with $f_{j}(\lambda) \geqslant 0$ when $\lambda>0$. This gives for $n \geqslant 0$

$$
\left|c_{n}^{\lambda}(x ; k)\right| \leqslant 1, \quad-1 \leqslant x \leqslant 1, \lambda \geqslant 0 .
$$

The inequality (4.14) does not hold when $\lambda<0$. To see this observe that

$$
c_{n k}^{\lambda}(\cos \theta ; k)=C_{n}^{\lambda}(\cos k \theta) / C_{n}^{\lambda}(1)
$$

follows from (4.8), and

$$
\left|C_{n}^{\lambda}(\cos \theta)\right| \leqslant C_{n}^{\lambda}(1)
$$

holds when $\lambda>0$ but not when $\lambda<0$ (see [21, Theorem 7.33.1]). This inequality fails for other $n$ 's as well. This can be shown for $n$ large by using Darboux's method on the generating function (see [21, §8.4]). It also fails for $n=k+1$, since

$$
c_{k+1}^{\lambda}(\cos \theta ; k)=\frac{(\lambda+1) \cos (k+1) \theta+\lambda \cos (k-1) \theta}{(2 \lambda+1)}
$$

Then,

$$
c_{k+1}^{\lambda}\left(\cos \frac{\pi}{k+1} ; k\right)=-1+\frac{2 \lambda}{2 \lambda+1} \cos \frac{(k-1) \pi}{2 k+2}<-1
$$

when $-1 / 2<\lambda<0$. It is likely to fail for all $n>k$ when $-1 / 2<\lambda<0$, but a proof will have to wait until we learn more about these polynomials.

5. Further facts. It is possible to evaluate $B_{n}^{\lambda}(x ; k)$ at a number of points, and then from (4.12) to evaluate $c_{n}^{\lambda}(x ; k)$ at the same points. One of these points arises naturally from an evaluation of the continuous $q$-ultraspherical polynomials given in [7]. These polynomials can be given by

$$
C_{n}(\cos \theta ; \beta \mid q)=\frac{(\beta ; q)_{n}}{(q ; q)_{n}} \sum_{k=0}^{n} \frac{\left(q^{-n} ; q\right)_{k}(\beta ; q)_{k}}{\left(q^{1-n} / \beta ; q\right)_{k}(q ; q)_{k}}\left(\frac{q}{\beta}\right)^{k} \exp i(n-2 k) \theta .
$$

When $\beta=\exp (2 i \theta)$ this gives

$$
C_{n}\left(\left(\beta^{1 / 2}+\beta^{-1 / 2}\right) / 2 ; \beta \mid q\right)=\beta^{-n / 2}\left(\beta^{2} ; q\right)_{n} /(q ; q)_{n},
$$


from Heine's formula

$$
{ }_{2} \Phi_{1}\left(a, \quad b ; q, \frac{c}{a b}\right)=\frac{(c / a ; q)_{\infty}(c / b ; q)_{\infty}}{(c ; q)_{\infty}(c / a b ; q)_{\infty}} .
$$

Setting $\beta=s^{\lambda k+1} \omega_{k}, q=s \omega_{k}$ and taking the limit as $s \rightarrow 1$ gives

$$
B_{m k+j}^{\lambda}(\cos \pi / k ; k)=\frac{(-1)^{m}(2 \lambda+1)_{m} \sin (j+1) \pi / k}{m ! \sin \pi / k},
$$

$j=0,1, \ldots, k-1 ; k \geqslant 2, m=0,2, \ldots$.

This can be extended. From the explicit formula (4.4)

$$
\begin{aligned}
B_{m k+j}^{\lambda}(\cos i \pi / k ; k) & =\sum_{s=0}^{m} U_{(m-s) k+j}(\cos i \pi / k) C_{s}(\cos i \pi) \\
& =\sum_{s=0}^{m} \frac{\sin [((m-s) k+j+1) i \pi / k]}{\sin i \pi / k} \frac{(2 \lambda)_{s}(-1)^{i s}}{s !} \\
& =\frac{(-1)^{m i} \sin (j+1) i \pi / k}{\sin i \pi / k} \sum_{s=0}^{m} \frac{(2 \lambda)_{s}}{s !} \\
& =(-1)^{m i} \frac{\sin (j+1) i \pi / k}{\sin i \pi / k} \frac{(2 \lambda+1)_{m}}{m !}
\end{aligned}
$$

for $i=1,2, \ldots, k-1 ; j=0,1, \ldots, k-1 ; k \geqslant 2 ; m=0,1 \ldots$ It is also possible to find the value when $x=1$. Again from the explicit formula (4.4)

$$
\begin{aligned}
B_{m k+j}(1 ; k)= & \sum_{s=0}^{m}[(m-s) k+j+1](2 \lambda)_{s} / s ! \\
= & \frac{(m k+j+1)(2 \lambda+1)_{m}}{m !}-k \sum_{s=1}^{m} \frac{(2 \lambda)_{s}}{(s-1) !} \\
= & \frac{(m k+j+1)(2 \lambda+1)_{m}}{m !}-\frac{2 \lambda k(2 \lambda+2)_{m-1}}{(m-1) !} \\
= & \frac{m k(2 \lambda+1)_{m}}{m !}+\frac{(j+1)(2 \lambda+1)_{m}}{m !} \\
& -\frac{(2 \lambda+1)^{m}(2 \lambda+2)_{m-1}}{m !}+\frac{m k(2 \lambda+2)_{m-1}}{m !} \\
= & \frac{(2 \lambda+1)_{m}\left[j+1+\frac{m k}{2 \lambda+1}\right],}{m !}[j
\end{aligned}
$$

where $j=0,1, \ldots, k-1 ; k \geqslant 1 ; m=0,1, \ldots$

By using these equations and (4.12) we obtain

$$
c_{m k+j}^{\lambda}(\cos i \pi / k ; k)=(-1)^{m i} \cos i j \pi / k,
$$

$i=0,1, \ldots, k ; j=0,1, \ldots, k-1, m=0,1, \ldots$.

The many monotonicity properties of the ultraspherical polynomials (see Szegö [21, Chapter 6]) are likely to extend to the sieved ultraspherical polynomials when they are considered on the intervals $(j-1) \pi / k \leqslant \theta \leqslant j \pi / k, j=1, \ldots, k$, and 
$x=\cos \theta$. In addition the graphs of the polynomials $p_{n}(x)$ and $p_{n+2 k}(x)$ are very similar for both $p_{n}(x)=c_{n}^{\lambda}(x ; k)$ and $p_{n}(x)=B_{n}^{\lambda}(x ; k) / B_{n}^{\lambda}(1 ; k)$. An extra arch has been added in each of the intervals $(j-1) \pi / k \leqslant \theta=\cos ^{-1}(x) \leqslant j \pi / k$ and the successive maxima and minima near $x=\cos i \pi / k$ seem to converge monotonically to their limits when the sequences $P_{2 m+j}(x)$ are considered as functions of $m=$ $0,1, \ldots$ with $j$ fixed.

One interesting consideration is to see how the point $x=\left(\beta^{1 / 2}+\beta^{-1 / 2}\right) / 2$ in (5.1), which is exterior to the interval $[-1,1]$, moves to $x=\cos (\pi / k)$, which is interior to $[-1,1]$, as $q$ approaches $\omega_{k}$. It is also interesting to see that the values of $c_{n}^{\lambda}(x ; k)$ are independent of $\lambda$ when $x=\cos (i \pi / k)$.

Rogers [19] found the linearization coefficients for the continuous $q$-ultraspherical polynomials:

$$
C_{n}(x ; \beta \mid q) C_{m}(x ; \beta \mid q)=\sum_{j=0}^{\min (m, n)} a(j, m, n) C_{m+n-2 j}(x ; \beta \mid q),
$$

where

$$
\begin{aligned}
a(j, m, n)= & \frac{(\beta q ; q)_{m+n-2 j}(q ; q)_{m+n-2 j}(\beta ; q)_{m-j}(\beta ; q)_{n-j}}{\left(\beta^{2} ; q\right)_{m+n-2 j}(\beta ; q)_{m+n-2 j}(q ; q)_{m-j}(q ; q)_{n-j}} \\
& \times \frac{(\beta ; q)_{j}\left(\beta^{2} ; q\right)_{m+n-j}}{(q ; q)_{j}(\beta q ; q)_{m+n-j}} .
\end{aligned}
$$

When $\beta=s^{\lambda k+1} \omega_{k}, q=s \omega_{k}$, it is easy to take the limit as $s \rightarrow 1$.

THEOREM 3. If $k=1,2, \ldots$, then

$$
B_{m}^{\lambda}(x ; k) B_{n}^{\lambda}(x ; k)=\sum_{j=0}^{\min (m, n)} a(j, m, n) B_{m+n-2 j}^{\lambda}(x ; k),
$$

with

$$
\begin{aligned}
a(j, m, n)= & \frac{(\lambda+1)_{\lfloor m+n+1-2 j / k\rfloor}(1)_{\lfloor m+n-2 j / k\rfloor}(\lambda+1)_{\lfloor m-j / k\rfloor}}{(2 \lambda+1)_{\lfloor m+n+1-2 j / k\rfloor}(\lambda+1)_{\lfloor m+n-2 j / k\rfloor}(1)_{\lfloor m-j / k\rfloor}} \\
& \times \frac{(\lambda+1)_{\lfloor n-j / k\rfloor}(\lambda+1)_{\lfloor j / k\rfloor}(2 \lambda+1)_{\lfloor m+n+1-j / k\rfloor}}{\left.(1)_{\lfloor n-j / k\rfloor}(1)_{\lfloor j / k\rfloor}(\lambda+1)_{\lfloor m+n+1-j / k}\right\rfloor}
\end{aligned}
$$

where in each of the subscripts, such as $\lfloor m-j / k\rfloor$, the integer $k$ divides all the integers before it.

The parenthesis that should be included was dropped to make the printing easier. Observe that $a(j, m, n) \geqslant 0$ when $\lambda>-1 / 2$.

A similar, but more complicated, formula holds for the sieved ultraspherical polynomials of the first kind. To see this rewrite (5.6) and (5.7) as

$$
c_{n}(x ; \beta \mid q) c_{m}(x ; \beta \mid q)=\sum_{j=0}^{\min (m, n)} b(j, m, n) c_{m+n-2 j}(x ; \beta \mid q)
$$


with $c_{n}(x ; \beta \mid q)=(q ; q)_{n} C_{n}(x ; \beta \mid q) /\left(\beta^{2} ; q\right)_{n}$ and

$$
\begin{aligned}
b(j, m, n)= & \frac{(1-\beta)\left(1-\beta q^{m+n-2 j}\right)\left(1-\beta^{2} q^{m}\right)\left(1-\beta^{2} q^{n}\right)}{(1+\beta)\left(1-\beta q^{m-j}\right)\left(1-\beta q^{n-j}\right)\left(1-\beta q^{j}\right)\left(1-\beta^{2} q^{m+n-j}\right)} \\
& \times \frac{(\beta q ; q)_{m-j}(q ; q)_{m}(\beta q ; q)_{n-j}(q ; q)_{n}(\beta q ; q)_{j}\left(\beta^{2} q ; q\right)_{m+n-j}}{(q ; q)_{m-j}\left(\beta^{2} q ; q\right)_{m}(q ; q)_{n-j}\left(\beta^{2} q ; q\right)_{n}(q ; q)_{j}(\beta q ; q)_{m+n-j}} .
\end{aligned}
$$

When $\beta=s^{\lambda k}, q=s \omega_{k}$ and $s \rightarrow 1$, the second group of factors, those involving $(a ; q)_{r}$, have a limit. The first also do but cases have to be considered. When $j \equiv m$ $(\bmod k)$ or $j \equiv n(\bmod k)$, then any zero factor in the bottom is obviously cancelled by one in the top. When $j \equiv(m+n)(\bmod k)$ this also happens, because $\omega_{k}^{j}=1$ and $\omega_{k}^{-j}=1$ are satisfied at the same time. When none of these conditions hold the only factor in the bottom that can vanish is $1-\beta q^{j}$ and this is cancelled by $1-\beta$. The resulting coefficients are too messy to state until necessary, but it is easy to see that the limiting coefficients $b(j, m, n)$ are nonnegative when $\lambda \geqslant 0$.

There are many other formulas that can be found, and they should be recorded somewhere. One example follows from an identity of Rogers [19]:

$$
C_{n}(x ; \gamma \mid q)=\sum_{j=0}^{\lfloor n / 2\rfloor} \frac{\beta^{j}(\gamma / \beta ; q)_{j}(\gamma ; q)_{n-j}\left(1-\beta q^{n-2 j}\right)}{(q ; q)_{j}(\beta q ; q)_{n-j}(1-\beta)} C_{n-2 j}(x ; \beta \mid q) .
$$

Set $\gamma=s^{\lambda k+1} \omega_{k}, \beta=s^{\mu k}, q=s \omega_{k}$ and take the limit as $s \rightarrow 1$. The result is

$$
B_{n}^{\lambda}(x ; k)=\sum_{j=0}^{\lfloor n / 2\rfloor} a(j, n) c_{n-2 j}^{\mu}(x ; k)
$$

with

$$
a(j, n)=\frac{2(\lambda+1-\mu)_{\lfloor j / k\rfloor}(\lambda+1)_{\lfloor n-j / k\rfloor}(2 \mu+1)_{\lfloor n-2 j / k\rfloor}}{(1)_{\lfloor j / k\rfloor}(\mu+1)_{\lfloor n-j / k\rfloor}(1)_{\lfloor n-2 j / k\rfloor}} b(j, n),
$$

where

$$
b(j, n)= \begin{cases}1 & \text { if } n-2 j \neq m k \\ (m+\mu) /(m+2 \mu) & \text { if } n-2 j=m k\end{cases}
$$

When $\gamma=s^{\lambda k}, \beta=s^{\mu k}, q=s \omega_{k}$ and $s \rightarrow 1$ the connection coefficients are found between $c_{n}^{\lambda}(x ; k)$ and $c_{j}^{\mu}(x ; k)$. It is also possible to connect two $B_{n}$ 's and to invert (5.13).

As we remarked, Rogers found (5.6), (5.7) and (5.12). In both cases he seems to have worked out the identity for low degree polynomials, guessed the answer and then proved it by induction. There is no way to do this for the sieved polynomials, since the formulas for the recurrence relation have too inany factors that are constant most of the time. It would be very hard and probably impossible to get enough data to guess the formula.

There is an inverse for both (5.6) and (5.13) (see [7]). We leave the inverse of (5.6) to others, or until we need it, and only give one special case of the inverse of (5.12). 
The $q$-identity is

$$
\begin{gathered}
w_{\beta}(x) C_{n}(x ; \beta \mid q)=\sum_{k=0}^{\infty} a(k, n) C_{n+2 k}(x ; \gamma \mid q) w_{\gamma}(x), \\
a(k, n)=\frac{\beta^{k}(\gamma / \beta ; q)_{k}\left(q^{n+1} ; q\right)_{2 k}\left(\gamma^{2} q^{n+2 k} ; q\right)_{\infty}\left(\beta q^{n+k+1} ; q\right)_{\infty}\left(1-\gamma q^{n+2 k}\right)(\beta ; q)_{\infty}}{(q ; q)_{k}\left(\gamma q^{n+k} ; q\right)_{\infty}\left(\beta^{2} q^{n} ; q\right)_{\infty}(\gamma ; q)_{\infty}}
\end{gathered}
$$

When $|\beta|<1,|q|<1$, the function $w_{\beta}(x)$ can be taken to be

$$
w_{\beta}(x)=\prod_{n=0}^{\infty} \frac{\left(1-2\left(2 x^{2}-1\right) q^{n}-q^{2 n}\right)}{\left(1-2\left(2 x^{2}-1\right) \beta q^{n}-\beta^{2} q^{2 n}\right)} .
$$

When $\gamma=q$, this simplifies to

$$
\frac{(q ; q)_{n}}{\left(\beta^{2} ; q\right)_{n}} w_{\beta}(x) C_{n}(x ; \beta \mid q)=A \sum_{j=0}^{\infty} \beta^{j} \frac{(q / \beta ; q)_{j}}{(q ; q)_{j}} \frac{(q ; q)_{n+j}}{(\beta q ; q)_{n+j}} 4\left(1-x^{2}\right) U_{n+2 j}(x)
$$

with

$$
A=\frac{(\beta ; q)_{\infty}(\beta q ; q)_{\infty}}{(q ; q)_{\infty}\left(\beta^{2} ; q\right)_{\infty}}
$$

Set $\beta=s^{\mu k}, q=s \omega_{k}$ and let $s \rightarrow 1$. The result is

$$
\begin{aligned}
& 2^{2(\mu-1)} \frac{|\sin k \theta|^{2 \mu}}{\sin \theta} c_{n}^{\mu}(\cos \theta ; k) \\
& =\frac{\Gamma(2 \mu)}{\Gamma(\mu) \Gamma(\mu+1)} \sum_{j=0}^{\infty} \frac{(1-\mu)_{\lfloor j / k\rfloor}(1)_{\lfloor(n+j) / k\rfloor}}{(1)_{\lfloor j / k\rfloor}(\mu+1)_{\lfloor(n+j) / k\rfloor}} \sin (n+2 j+1) \theta .
\end{aligned}
$$

Formulas (5.15) and (5.16) are the explicit forms of the identities Allaway showed existed in his Ph.D. thesis [2].

6. Open problems. Many problems suggest themselves for these polynomials. One is to show that the Poisson kernel for these series is positive for $-1<r<1$ when $\lambda>-1 / 2$. This can probably be done using an expression for the Poisson kernel for the continuous $q$-ultraspherical polynomials due to Gasper and Rahman [16]. However, their formula will be an integral representation for the Poisson kernel and so may only hold for $\lambda>0$ for the polynomials of the first kind. Thus there may be a need to find a series representation for the Poisson kernel similar to Bailey's [11] that will give the positivity.

A potentially very important result would be the second order differential equations these polynomials satisfy. See Atkinson and Everitt [10] for a proof of the existence of these equations. It may be possible to derive them from the $q$-divided difference equation in [9].

It would also be very interesting to see how much of the qualitative behaviour can be extended to general orthogonal polynomials. A start on this when the recurrence relation has the form

$$
x p_{n}(x)=a_{n} p_{n+1}(x)+b_{n} p_{n}(x)+a_{n-1} p_{n-1}(x)
$$


with

$$
\begin{aligned}
& b_{n}=(-1)^{n} b / n+O\left(1 / n^{2}\right), \\
& a_{n}=1 / 2+(-1)^{n} a / n+O\left(1 / n^{2}\right)
\end{aligned}
$$

was made by Nevai [18]. If $p_{n}(x)$ are orthogonal with respect to an absolutely continuous measure on $[-1,1]$, say $W(x)$, then Nevai showed that

$$
W(x) \geqslant A|x|^{c}\left(1-x^{2}\right)^{d}, \quad-1<x<1,
$$

for some $c, d>-1$. Actually he proved more, but for a complete statement of his results see his paper. It is likely that a similar theorem is true with respect to a measure

$$
\prod_{j=0}^{k}|x-\cos (\pi j / k)|^{\alpha(j)},
$$

when the recurrence coefficients have an appropriate behaviour in the subsequence $a_{n k+j}, j=0,1, \ldots, k-1$. However it is not clear yet whether it is better to take the orthonormal polynomials as Nevai did, or take another normalization as we did in this paper. Probably there are theorems in both cases.

An extension of the continuous $q$-ultraspherical polynomials was considered in [8]. It should be very interesting to let $q$ approach a root of unity and find the orthogonality relation that arises. This will give some extensions of the polynomials of Pollaczek.

\section{REFERENCES}

1. W. Al-Salam, Wm. R. Allaway and R. Askey, A characterization of the continuous q-ultraspherical polynomials (to appear).

2. Wm. R. Allaway, The identification of a class of orthogonal polynomials, Ph.D. thesis, University of Alberta, Canada, 1972.

3. G. E. Andrews, The theory of partitions, Addison-Wesley, Reading, Mass., 1977.

4. R. Askey, The q-gamma and q-beta functions, Applicable Analysis 8 (1978), 125-141.

5. ___ Ramanujan's extensions of the gamma and beta functions, Amer. Math. Monthly 87 (1980), 346-359.

6. R. Askey and M. Ismail, The Rogers q-ultraspherical polynomials, Approximation Theory. III (E. W. Cheney, ed.), Academic Press, New York, 1980, pp. 175-182.

7. _ A generalization of the ultraspherical polynomials, Studies in Pure Mathematics (P. Erdös, ed.), Birkhäuser, Basel, 1983, pp. 55-678.

8. __ Recurrence relations, continued fractions and orthogonal polynomials (to appear).

9. R. Askey and J. Wilson, Some basic hypergeometric orthogonal polynomials that generalize Jacobi polynomials, Mem. Amer. Math. Soc. (to appear).

10. F. V. Atkinson and W. N. Everitt, Orthogonal polynomials which satisfy second order differential equations (E. B. Christoffel, P. L. Butzer and F. Fehér, eds.), Birkhäuser, Basel, 1981, pp. 173-181.

11. W. N. Bailey, The generating function of Jacobi polynomials, J. London Math. Soc. 13 (1938), 8-12.

12. __ Generalized hypergeometric series, Hafner, New York, 1972.

13. D. Bressoud, Theta function identities and orthogonal polynomials, Analytic Number Theory (Proceedings, Philadelphia, Pa., 1980), Lecture Notes in Math., vol. 899, Springer-Verlag, Berlin, Heidelberg and New York, 1981.

14. T. S. Chihara, An introduction to orthogonal polynomials, Gordon \& Breach, New York, 1978.

15. E. Feldheim, Sur les polynomes géneralisés de Legendre, Izv. Akad. Nauk SSSR Ser. Mat. 5 (1941), 241-248; Russian transl., ibid, 248-254.

16. G. Gasper and M. Rahman, Positivity of the Poisson kernel for the continuous $q$-ultraspherical polynomials, SIAM J. Math. Anal. 14 (1983), 409-420. 
17. I. J. Lanzewizky, Über die orthogonalität Fejér Szegöschen polynome, C. R. (Dokl.) Acad. Sci. URSS 31 (1941), 199-200.

18. P. Nevai, Orthogonal polynomials defined by a recurrence relation, Trans. Amer. Math. Soc. 250 (1979), 369-384.

19. L. J. Rogers, Third memoir on the expansion of certain infinite products, Proc. London Math. Soc. 26 (1895), 15-32.

20. L. J. Slater, Generalized hypergeometric functions, Cambridge Univ. Press, Cambridge, 1966.

21. G. Szegö, Orthogonal polynomials, 4th ed., Amer. Math. Soc. Colloq. Publ., vol. 23, Amer. Math. Soc., Providence, R.I., 1975.

Department of Mathematics, University of Alberta, Edmonton, Alberta, Canada T6G 2G1

Department of Mathematical Sciences, Lakehead University, Thunder Bay, Ontario, Canada P7B 5E1

Department of Mathematics, University of Wisconsin, Madison, Wisconsin 53706 